\title{
Prediction of ICU admission after orthopedic surgery in elderly patients
}

\author{
Yongzhong Tang ${ }^{1}$, Hao $\mathrm{Li}^{2}$, Ziyi Guo
}

\begin{abstract}
Objectives: Prediction of ICU admission after surgery are important for rational decision-making for different patients in clinical practice. Little information is available about the risk factors of postoperative ICU admission in elderly patients undergoing orthopedic surgery. This study aimed to identify risk factors and develop a predictive model for postoperative ICU admission in elderly patients undergoing orthopedic surgery.

Methods: A total of 2826 cases of elderly patients receiving orthopedic surgery from October 2010 to September 2016 were retrospectively collected and analyzed. Logistic regression was used to evaluate the impacts of covariates. Support vector machine (SVM) was employed to develop a predictive model based on all pre-operative covariates and the demographic information.

Results: There were 256 patients transferred to ICU after surgery. ASA III or IV and emergency surgery were found to be independent risk factors while neuraxial anesthesia and joint surgery were protective factors. In addition, a SVM-based predictive model was developed, which had a sensitivity of $90.99 \%$, a specificity of $99.10 \%$ and an area under ROC curve of 0.9678 .

Conclusions: Our study revealed that emergency surgery, anesthesia method, surgery type and ASA grade were risk factors to predict postoperative ICU admission in elderly orthopedic patients.
\end{abstract}

KEYWORDS: Orthopedic surgery, Elderly patients, ICU admission.

doi: https://doi.org/10.12669/pjms.37.4.3371

How to cite this:

Tang Y, Li H, Guo Z. Prediction of ICU admission after orthopedic surgery in elderly patients. Pak J Med Sci. 2021;37(4):1179-1184. doi: https://doi.org/10.12669/pjms.37.4.3371

This is an Open Access article distributed under the terms of the Creative Commons Attribution License (http://creativecommons.org/licenses/by/3.0), which permits unrestricted use, distribution, and reproduction in any medium, provided the original work is properly cited.

1. Dr. Yongzhong Tang, MD.

Department of Anesthesiology, Third Xiangya Hospital,

Central South University, Changsha, China.

2. Dr. Hao Li, MD.

Intensive Care Unit,

Taikang Xianlin Drum Tower Hospital, Nanjing, China.

3. Dr. Ziyi Guo, MM.

Department of Orthopedic Surgery,

Shulan (Hangzhou) Hospital, Hangzhou, China.

Department of Orthopedic Surgery,

First Affiliated Hospital of Zhejiang University,

Hangzhou, China.

Note: Yongzhong Tang and Hao Li contributed equally to this study.

Correspondence:

Dr. Ziyi Guo, MM.

No. 848 Dongxin Road,

Hangzhou 310000, China.

Email: ziyiguo_dr@163.com

* Received for Publication:

* Revision Received:

* Revision Accepted:
July 26, 2020

March 10, 2021

March 25, 2021

\section{INTRODUCTION}

Surgery is a common treatment method for fractures. ${ }^{1}$ However, elderly patients are frequently associated with many preoperative comorbidities ${ }^{2}$ or may have reduced organ functions, resulting in high complications rates, ${ }^{3,4}$ high postoperative mortalities and impaired quality of life., Admission to Intensive Care Unit (ICU) for those elderly patients, will not only lead to high medical $\operatorname{costs}^{7}$ and heavy medical burdens ${ }^{8}$, but also poor prognosis.

It is important to balance the benefit and harm of the surgery and to choose the timing of the surgery. Some studies have shown ${ }^{9}$ that 90 -day overall mortality after hip surgery in elderly patients may be related to the posterior surgical approach, anesthesia technique, and the use of anticoagulant. However, it seems surgical timing affected only 
by holidays or weekends doses not increase the mortality rates, ${ }^{10}$ and early resection of elbow joint heterotopic ossification was conducive to rehabilitation..$^{11}$ Therefore, for proper allocation of medical resource and implementation of rational treatment decision, it is important to identify the risk factors associated with the postoperative ICU admission. Especially, an ICU admission predictive model suitable for elderly is still needed.

In the current studies, preoperative and intraoperative risk factors were analyzed. ${ }^{12,13} \mathrm{We}$ collected both perioperative and intraoperative data of elderly patients receiving orthopedic surgery and successfully established a model for predicting postoperative admission to ICU with a supervised machine learning approach.

\section{METHODS}

This study was approved by the Ethics Committee of the Third Xiangya Hospital of Central South University (No.2018-S221). Written consent was obtained from each patient. A total of 2826 elderly patients (aged 60-100 years) receiving orthopedic surgeries in the Third Xiangya Hospital, a tertiary-care college hospital, from October 2010 to September 2016 were retrospectively analyzed, among which 256 were postoperatively admitted to ICU. Both elective and emergency surgery were included. The exclusion criteria were reoperation due to surgery complications, preoperative ICU stay, severe cardio/pulmonary disease and using cardiovascular active drug prior to surgery.
All collected data were exported from the Hospital Information System (HIS) and Anesthesia Information Management System of the Third Xiangya Hospital (Fig.1) via its embedded data portal under the supervision of a professional engineer. Entries to collect information were selected and the corresponding records of the patients were automatically exported and manually reviewed. The following data were collected:

1. General information including age, gender, BMI, smoking history, alcohol intake and hospital stay.

2. Preoperative concurrent clinical diagnosis including hypertension, diabetes, coronary heart disease, peripheral vascular diseases, heart failure, pneumonia, and stroke.

3. Surgery-related information including operation types, surgical duration, total influid amount, bleeding volume, and transfusion volume.

4. Anesthesia-related information including anesthesia method and ASA status.

5. ICU admission status after surgery.

6. Laboratory testing results within one week before surgery. Manual cross-check was performed by two doctors and further examined by a senior doctor when there were discrepancies.

For categorical variables, frequency distributions and two-way classification tables were present and Fisher's exact test or Chi-square test was used for comparison. For continuous variables, means,

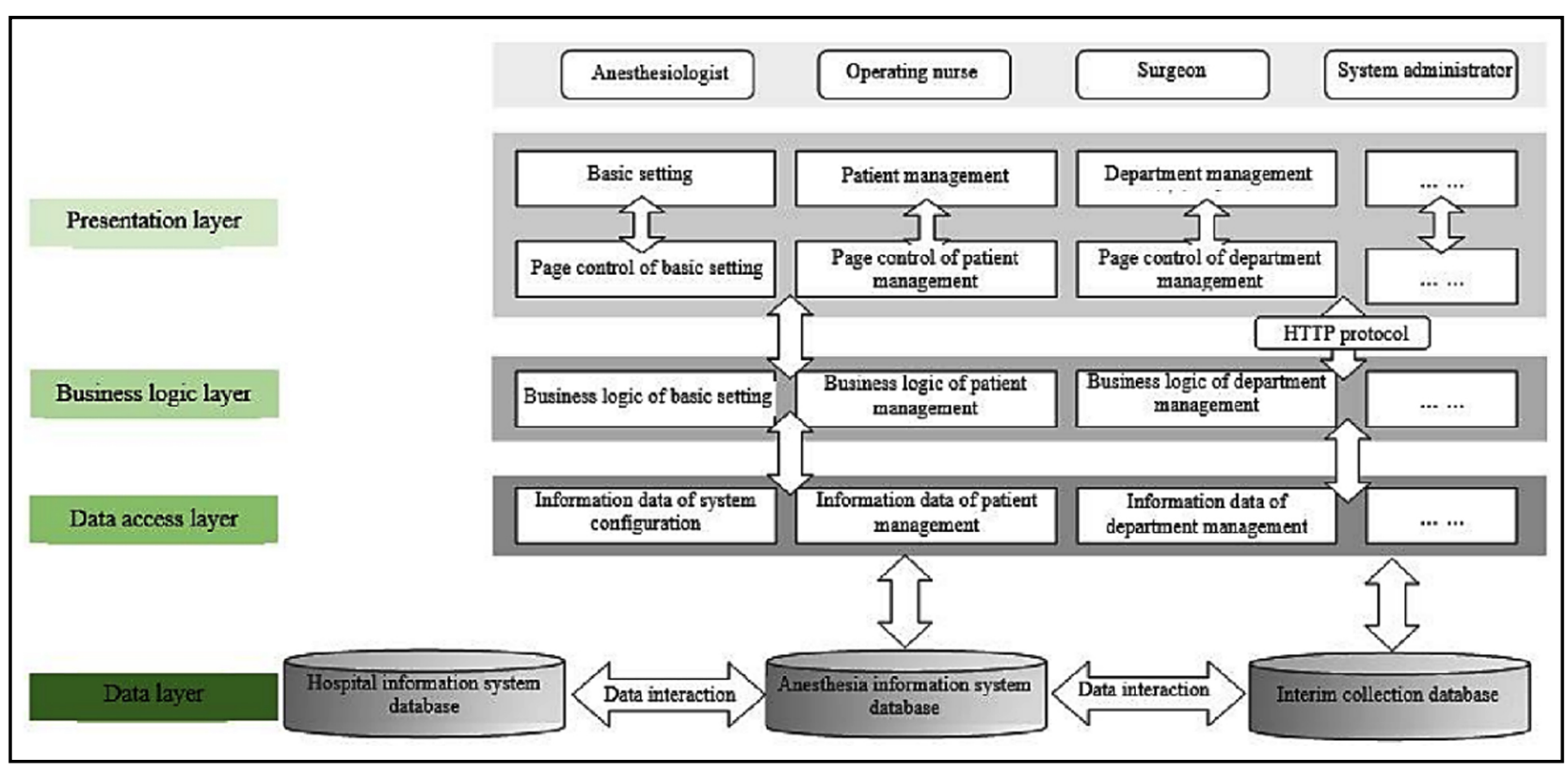

Fig.1: Hospital information systems instruction. 
Table-I: Univariate analysis of continuous variables of the patients with and without postoperative ICU admission.

\begin{tabular}{lccccc}
\hline & \multicolumn{3}{c}{ Postoperative ICU admission } \\
\cline { 2 - 4 } & \multicolumn{2}{c}{ No $(n=2570)$} & Yes $(n=256)$ & \\
\cline { 2 - 5 } & Mean & SD & Mean & \\
\hline Age (years) & 68.51 & 8.25 & 69.8 & 7.36 & 0.0338 \\
BMI & 23.03 & 3.66 & 22.75 & 3.42 & 0.4631 \\
Height (cm) & 161.5 & 7.31 & 163.31 & 7.28 & 0.0976 \\
Weight (kg) & 59.06 & 10.78 & 60.79 & 10.45 & 0.0446 \\
Min preoperation hemoglobin (g/L) & 120.46 & 21.21 & 116.61 & 24.61 & 0.0086 \\
Min preoperation calcium (mmol/L) & 2.25 & 0.17 & 2.2 & 0.17 & 0.0002 \\
Min preoperation potassium (mmol/L) & 3.98 & 0.45 & 3.92 & 0.44 & 0.0184 \\
Min preoperation albumin (g/L) & 39.12 & 5.09 & 36.24 & 6.39 & 0.0000 \\
Max preoperation neutrophil count (109/L) & 5.87 & 3.39 & 7.23 & 4.68 & 0.0001 \\
Total influid volume (ml) & 1636.97 & 1209.03 & 1943.86 & 1463.19 & 0.0010 \\
Transfusion volume (ml) & 256.05 & 566.21 & 394.49 & 793.18 & 0.0146 \\
Urine (ml) & 565.53 & 545.19 & 604.01 & 596.31 & 0.4524 \\
Surgery duration (mins) & 158.56 & 87.25 & 186.9 & 109.77 \\
\hline
\end{tabular}

Note that each of the variables was analyzed alone and the joint effects with other variables were not considered. SD, standard deviations.

standard deviations, and ranges were provided. T-test or ANOVA (or Mann-Whitney's test or Kruskal Wallis test, as appropriate) was used to compare the mean values. Logistic regression was utilized to evaluate the impacts of covariates. The prediction model was developed with support vector machine. A two-tailed $\mathrm{p}<0.05$ was considered statistically significant. All analyses were performed with $\mathrm{R}$ (v3.4.1) and its packages pROC (1.10.0) and kernlab (0.9-25).

\section{RESULTS}

A total of 2826 patients were enrolled. After selection, 115 patients with insufficient data (with $>30 \%$ missing) were excluded. The included patients were divided into two groups for further analysis according to postoperative ICU admission status. The characteristics of the two groups are listed in Table-I. Patients with and without postoperative ICU admission displayed significant difference in many variables (Tables-I \& II). Of note, gender, hypertension, surgery type, anesthesia method, mechanical ventilation, and emergency surgery were significantly associated with postoperative ICU admission. By fitting a logistic regression model including all covariates, we further found that five variables were significant factors (Table-III). We saw that patients with ASA III or IV had higher risk than those with ASA I-II; that joint surgery had lower risk than trauma surgery, that neuraxial anesthesia had lower risk than the other three types; and that emergency surgery had higher risk than elective surgery.

Next, we developed a model to predict ICU admission in elderly patients after orthopedic surgery with support vector machine method using the default type "C-svc" on all pre-operative covariates and the demographic information. The prediction model had a sensitivity of $90.99 \%$, a

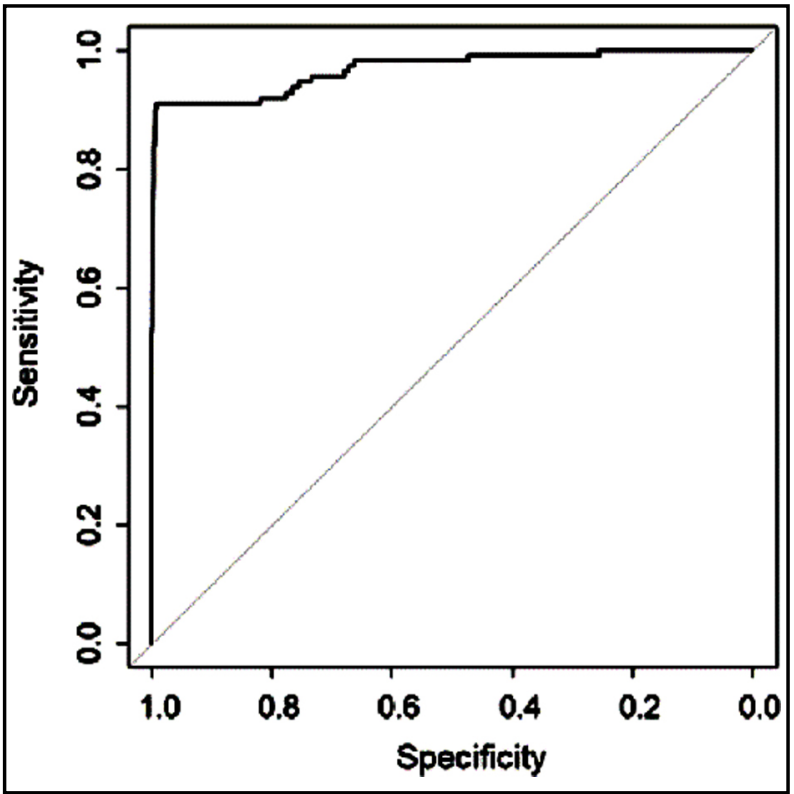

Fig.2: Receiver operating characteristic (ROC) curve for the prediction of postoperative ICU admission in elderly patients undergoing orthopedic surgery. 
Yongzhong Tang et al.

Table-II: Univariate analysis of categorical variables of the patients with and without postoperative ICU admission.

\begin{tabular}{|c|c|c|c|c|c|c|}
\hline & & \multicolumn{4}{|c|}{ Postoperative ICU admission } & \multirow[b]{3}{*}{$p$} \\
\hline & & \multicolumn{2}{|c|}{ No $(n=2570)$} & \multicolumn{2}{|c|}{ Yes $(n=256)$} & \\
\hline & & Frequency & $\%$ & Frequency & $\%$ & \\
\hline \multirow[t]{2}{*}{ Gender } & $\mathrm{F}$ & 1343 & 52.26 & 90 & 35.16 & $1.86 \mathrm{E}-07$ \\
\hline & $\mathrm{M}$ & 1227 & 47.74 & 166 & 64.84 & \\
\hline \multirow[t]{2}{*}{ Smoking } & No & 2336 & 90.89 & 236 & 92.19 & 0.5669 \\
\hline & Yes & 234 & 9.11 & 20 & 7.81 & \\
\hline \multirow[t]{2}{*}{ Alcohol intake } & No & 2430 & 94.55 & 247 & 96.48 & 0.2392 \\
\hline & Yes & 140 & 5.45 & 9 & 3.52 & \\
\hline \multirow[t]{3}{*}{ ASA } & I\&II & 1336 & 59.12 & 90 & 52.63 & 0.1489 \\
\hline & III & 856 & 37.88 & 73 & 42.69 & \\
\hline & IV & 68 & 3.01 & 8 & 4.68 & \\
\hline \multirow[t]{2}{*}{ Preoperative pneumonia } & No & 2361 & 91.87 & 235 & 91.8 & 0.9051 \\
\hline & Yes & 209 & 8.13 & 21 & 8.2 & \\
\hline \multirow[t]{2}{*}{ Hypertension } & No & 1980 & 77.04 & 216 & 84.38 & 0.0072 \\
\hline & Yes & 590 & 22.96 & 40 & 15.62 & \\
\hline \multirow[t]{2}{*}{ Stroke } & No & 2496 & 97.12 & 252 & 98.44 & 0.3047 \\
\hline & Yes & 74 & 2.88 & 4 & 1.56 & \\
\hline \multirow[t]{2}{*}{ Coronary heart disease } & No & 2399 & 93.35 & 239 & 93.36 & 1 \\
\hline & Yes & 171 & 6.65 & 17 & 6.64 & \\
\hline \multirow[t]{2}{*}{ Chronic heart failure } & No & 2560 & 99.61 & 254 & 99.22 & 0.6773 \\
\hline & Yes & 10 & 0.39 & 2 & 0.78 & \\
\hline \multirow[t]{2}{*}{ Chronic kidney disease } & No & 2516 & 97.9 & 252 & 98.44 & 0.7274 \\
\hline & Yes & 54 & 2.1 & 4 & 1.56 & \\
\hline \multirow[t]{2}{*}{ Hyperlipidemia } & No & 2561 & 99.65 & 255 & 99.61 & 1 \\
\hline & Yes & 9 & 0.35 & 1 & 0.39 & \\
\hline \multirow[t]{6}{*}{ Surgery classification } & 1 & 730 & 28.4 & 114 & 44.53 & $1.40 \mathrm{E}-10$ \\
\hline & 2 & 829 & 32.26 & 94 & 36.72 & \\
\hline & 3 & 906 & 35.25 & 35 & 13.67 & \\
\hline & 4 & 27 & 1.05 & 3 & 1.17 & \\
\hline & 5 & 55 & 2.14 & 7 & 2.73 & \\
\hline & 6 & 23 & 0.89 & 3 & 1.17 & \\
\hline \multirow[t]{4}{*}{ Anesthesia method } & GA & 1119 & 43.54 & 158 & 61.72 & 4.26E-08 \\
\hline & IA & 16 & 0.62 & 4 & 1.56 & \\
\hline & NB & 171 & 6.65 & 16 & 6.25 & \\
\hline & NA & 1264 & 49.18 & 78 & 30.47 & \\
\hline \multirow[t]{2}{*}{ Mechanical ventilation } & No & 1451 & 56.46 & 98 & 38.28 & 3.59E-08 \\
\hline & Yes & 1119 & 43.54 & 158 & 61.72 & \\
\hline \multirow[t]{2}{*}{ Emergency surgery } & No & 2293 & 92.95 & 169 & 67.87 & 4.19E-27 \\
\hline & Yes & 174 & 7.05 & 80 & 32.13 & \\
\hline
\end{tabular}

Surgery Classification: 1=Trauma, 2=Spinal, 3=Joint, 4=Microsurgery, 5=Tumor, 6=Plastic.

Anesthesia Method: GA=General with mechanical ventilation, NB=Nerve Blocking,

$\mathrm{NA}=$ Neuraxial Anesthesia, IA =General without mechanical ventilation.

specificity of $99.10 \%$ and an area under the ROC curve of 0.9678 (Fig.2).

\section{DISCUSSION}

Orthopedic surgeries, especially hip and knee replacements, in elderly patients have received extensive attention from medical communities. Both the United States and Europe have
Table-III: Risk factors for postoperative ICU admission.

\begin{tabular}{lccc}
\hline & Odds ratio & $95 \%$ CI & $p$ \\
\hline ASA=III & 6.01 & $(1.4,25.76)$ & 0.0157 \\
ASA=IV & 250.75 & $(5.58,11266.39)$ & 0.0044 \\
Joint surgery & 0.13 & $(0.02,0.71)$ & 0.0192 \\
Neuraxial Anesthesia & 0.25 & $(0.08,0.73)$ & 0.0119 \\
Emergency & 5.1 & $(1.8,14.41)$ & 0.0021 \\
Gender (Male) & 1.6200 & $(0.95,2.75)$ & 0.0750 \\
\hline
\end{tabular}

CI, confidence interval. 
established hip replacement registries ${ }^{9,14}$ to find evidence-based criteria for optimal perioperative management approaches. Previous studies ${ }^{9}$ have shown that posterior approach surgery could cause less injury to tissues that mechanical ventilation was not recommended for spinal anesthesia to protect respiratory function and that anticoagulant drugs could be used to reduce the formation of postoperative thrombosis and protect the blood supply of the postoperative hip joint without increasing the risk of postoperative hemorrhage. This study aimed to predict postoperative ICU admission in elderly patients undergoing orthopedic surgery.

We found that ASA III or IV and emergency surgery were independent risk factors while neuraxial anesthesia and joint surgery were protective factors. ASA III or IV was found to be significantly associated with postoperative ICU admission. The ASA classification is an assessment of patient's preoperative pathophysiological status $^{3}$ that can predict the perioperative mortality of patients and was hence included for analysis in this study. Patients with ASA III or IV has been reported to have a perioperative mortality rate of $1.8-23 \%{ }^{15}$ Patients receiving emergency surgeries are often accompanied by multiple system and organ injuries. The urgency of surgery and the complexity of disease ${ }^{16}$ often result in lack of sufficient testing and examination, making it difficult for the surgeons and anesthesiologists to conduct adequate assessment of patients and comprehensively grasp the disease conditions. The risk of anesthesia is therefore increased. In addition, emergency surgery itself is also an independent risk factor of ASA risk classification. Neuraxial anesthesia does not require mechanical ventilation. During the operation, the spontaneous breathing of patient is usually maintained, pulmonary functions are thereby protected, the injury to alveolar mucosa caused by mechanical ventilation are reduced, and the incidence of postoperative ventilator-associated pulmonary atelectasis and pneumonia ${ }^{17}$ is reduced. These provide favorable conditions for patients to be admitted to general wards after surgery, and also improve the overall conditions of the patients, shorten bed-rest time, facilitate early ambulation, and further reduce the incidence of transferring from general ward to ICU after surgery. Joint surgery is less likely to cause ICU admission than trauma surgery. Most joint surgeries are operated in aseptic and clean condition, resulting in low incidence of serious infections and consequent respiratory and circulatory complications. ${ }^{18}$ In addition, joint orthopedic surgeries usually are not accompanied by other organ injuries. By contrast, trauma surgeries were conducted in traumatic orthopedic wounds. They often require emergency treatment ${ }^{19}$ and have higher incidence rates of peptic ulcer. ${ }^{20}$

Female was a protective factor in many studies for estrogen. Several researches have reported that estrogen has protective effects against trauma and inflammation, ${ }^{21-23}$ but in this study gender was a detrimental factor that needs to be further investigated (Table-III). For female patients over 60 years, estrogen level may not have sufficient contribution, since the OR=1.62 (95\% CI: 0.95-2.75) of male gender did not show statistical significance $(\mathrm{p}=0.075)$.

The possibility of ICU admission rises with the increase of age. Elderly patients have increased incidences of preoperative comorbidities, ${ }^{2}$ risks of microthrombosis ${ }^{24}$ and postoperative pulmonary function injury. Thus, it is important to assess the possibility of postoperative ICU admission in elderly patients. Here we found that among the elderly population, age was not a significant predictor for postoperative ICU admission, which suggests patient conditions might play a more important role.

A precision ICU admission model for elderly patients after orthopedic surgery is important for the doctors to make proper decisions. Our model can be easily implemented as it does not require complex variables, and the supervised machine learning approach resulted in a satisfying accuracy. The ICU admission model had a better specificity and sensitivity than the previous prediction models such as POSSIUM and P-POSSIUM. 25,26

Limitation of the study: This study has limitations. With a single-center cohort, this study may have bias, therefore future multi-center studies are required to generalization the results of our study.

\section{CONCLUSIONS}

Our SVM-based prediction model of ICU admission in elderly patients after orthopedic surgery has high specificity and sensitivity, which provides surgeons and anesthesiologists a tool to evaluate the benefit and harm and determine the timing of the surgery.

Acknowledgements: We thank Shanghai Ruihui Biotech for supports in statistical analysis. 
Grant Support \& Financial Disclosures: None.

Conflicts of Interest: None.

\section{REFERENCES}

1. Einhorn TA, Gerstenfeld LC. Fracture healing: mechanisms and interventions. Nat Rev Rheumatol. 2015;11(1):45-54. doi: 10.1038/nrrheum.2014.164

2. Shimada S, Sawada N, Oae S, Seki J, Takano Y, Ishiyama $Y$, et al. Safety and curability of laparoscopic gastrectomy in elderly patients with gastric cancer. Surg Endosc. 2018;32:4277-4283. doi: 10.1007/s00464-018-6177-1

3. Kempenaers K, Van Calster B, Vandoren C, Sermon A, Metsemakers WJ, Vanderschot P, et al. Are the current guidelines for surgical delay in hip fractures too rigid? A single center assessment of mortality and economics. Injury. 2018;49:1169-1175. doi: 10.1016/j.injury.2018.03.032

4. Peacock O, Bassett MG, Kuryba A, Walker K, Davies $\mathrm{E}$, Anderson I, et al. Thirty-day mortality in patients undergoing laparotomy for small bowel obstruction. Br J Surg. 2018;105:1006-1013. doi: 10.1002/bjs.10812

5. Tang B, Green C, Yeoh AC, Husain F, Subramaniam A. Post-operative outcomes in older patients: A singlecentre observational study. ANZ J Surg. 2018;88:421-427. doi: 10.1111/ans.14433

6. Wang $\mathrm{X}, \mathrm{Hu} \mathrm{Y}, \mathrm{Zhao} \mathrm{B}, \mathrm{Su} \mathrm{Y}$. Predictive validity of the ACS-NSQIP surgical risk calculator in geriatric patients undergoing lumbar surgery. Medicine (Baltimore). 2017;96(43):e8416. doi: 10.1097/MD.0000000000008416

7. Mahmoudian-Dehkordi A, Sadat S. A Generic Simulation Model of the Relative Cost-Effectiveness of ICU Versus Step-Down (IMCU) Expansion. J Intensive Care Med. 2017;35:191-202. doi: 10.1177/0885066617737303

8. Angus DC, Barnato AE, Linde-Zwirble WT, Weissfeld LA, Watson RS, Rickert T, et al. Use of intensive care at the end of life in the United States: An epidemiologic study. Crit Care Med. 2004;32(3):638-643. doi: 10.1097/01. ccm.0000114816.62331.08

9. Hunt LP, Ben-Shlomo Y, Clark EM, Dieppe P, Judge A, MacGregor AJ, et al. 90-day mortality after 409,096 total hip replacements for osteoarthritis, from the National Joint Registry for England and Wales: a retrospective analysis. Lancet. 2013;382(9898):1097-1104. doi: 10.1016/S01406736(13)61749-3

10. Wong SHJ, Fang XC, Yee KHD, Wong TM, Pun CTT, Lau $\mathrm{TW}$, et al. Hip fracture time-to-surgery and mortality revisited: mitigating comorbidity confounding by effect of holidays on surgical timing. Int Orthop. 2018;42:1789-1794. doi: 10.1007/s00264-017-3737-2

11. He SK, Yi M, Zhong G, Cen SQ, Chen JL, Huang FG. Appropriate excision time of heterotopic ossification in elbow caused by trauma. Acta Orthop Traumatol Turc. 2018;52(1):27-31. doi: 10.1016/j.aott.2017.11.008

12. Lamberts MP. Indications of cholecystectomy in gallstone disease. Curr Opin Gastroenterol. 2018;34(2):97-102. doi: 10.1097/MOG.0000000000000419

13. Sura K, Grills IS, Vu CC, Stevens CW, Ye H, Guerrero TM. Improved Survival With Increased Time-ToRadiation and Sequential Chemotherapy After Surgery for pN2 Non-Small-cell Lung Cancer. Clin Lung Cancer. 2018;19(2):e185-e194. doi: 10.1016/j.cllc.2017.10.011

14. Kelly MP, Prentice HA, Wang W, Fasig BH, Sheth DS, Paxton EW. Reasons for Ninety-Day Emergency Visits and Readmissions After Elective Total Joint Arthroplasty: Results From a US Integrated Healthcare System. J Arthroplasty. 2018;33:2075-2081. doi: 10.1016/j.arth.2018.02.010
15. Pearse RM, Moreno RP, Bauer P, Pelosi P, Metnitz P, Spies $\mathrm{C}$, et al. Mortality after surgery in Europe: a 7 day cohort study. Lancet. 2012;380(9847):1059-1065. doi: 10.1016/ S0140-6736(12)61148-9

16. Kim B, Jeong H, Kim J, Kim T, Kim K, Lee H, et al. Incidence and risk factors of delayed intracranial hemorrhage in the emergency department. Am J Emerg Med. 2018;36(2):271-276. doi: 10.1016/j.ajem.2017.08.009

17. Moraes L, Silva PL, Thompson A, Santos CL, Santos RS, Fernandes MVS, et al. Impact of Different Tidal Volume Levels at Low Mechanical Power on Ventilator-Induced Lung Injury in Rats. Front Physiol. 2018;9:318. doi: 10.3389 /fphys.2018.00318

18. Yen SH, Chen JH, Lu YD, Wang JW. Perioperative Complications of Total Knee Arthroplasty in Dialysis Patients. J Arthroplasty. 2018;33(3):872-877. doi: 10.1016/j. arth.2017.09.058

19. Weber CD, Hildebrand F, Kobbe P, Lefering R, Sellei RM, Pape HC, et al. Epidemiology of open tibia fractures in a population-based database: update on current risk factors and clinical implications. Eur J Trauma Emerg Surg. 2019;45:445-453. doi: 10.1007/s00068-018-0916-9

20. Barash PG CB, Stoelting RK, Cahalan M, Stock MC. Clinical Anesthesia, 6th edition Philadelphia, Lippincott Williams and Wilkins. 2009.

21. Pedersen AL, Brownrout JL, Saldanha CJ. Neuroinflammation and neurosteroidogenesis: Reciprocal modulation during injury to the adult zebra finch brain. Physiol Behav. 2018;187:51-56. doi: 10.1016/j. physbeh.2017.10.013

22. Jardi F, Laurent MR, Claessens F, Vanderschueren D. Estradiol and Age-Related Bone Loss in Men. Physiol Rev. 2018;98(1):1. doi: 10.1152/ physrev.00051.2017

23. Horng $\mathrm{HC}$, Chang $\mathrm{WH}$, Yeh $\mathrm{CC}$, Huang BS, Chang $\mathrm{CP}$, Chen YJ, et al. Estrogen Effects on Wound Healing. Int J Mol Sci. 2017;18:2325. doi: 10.3390/ijms18112325

24. Karcutskie CA, Meizoso JP, Ray JJ, Horkan D, Ruiz $\mathrm{XD}$, Schulman CI, et al. Association of Mechanism of Injury With Risk for Venous Thromboembolism After Trauma. JAMA Surg. 2017;152(1):35-40. doi: 10.1001/ jamasurg.2016.3116

25. Cao Y, Bass GA, Ahl R, Pourlotfi A, Geijer H, Montgomery $\mathrm{S}$, et al. The statistical importance of P-POSSUM scores for predicting mortality after emergency laparotomy in geriatric patients. BMC Med Inform Decis Mak. 2020;20(1):86. doi: 10.1186/s12911-020-1100-9

26. Simpson G, Parker A, Hopley P, Wilson J, Magee C. Pre-operative psoas major measurement compared to P-POSSUM as a prognostic indicator in over-80s undergoing emergency laparotomy. Eur J Trauma Emerg Surg. 2020;46(1):215-220. doi: 10.1007/s00068-018-1025-5

\section{Authors' Contribution:}

ZG: Conceived and designed the study, did manuscript writing and is responsible for integrity of the study.

YT \& HLJ: Did data collection and statistical analysis \& editing of manuscript.

All authors did review and final approval of manuscript. 